\title{
AVALIAÇÃO MORFOLÓGICA E QUANTITATIVA DOS ÁCINOS MUCOSOS DA GLÂNDULA SUBLINGUAL DE RATOS COM DIABETES CRÔNICO E SUPLEMENTADOS COM QUERCETINA
}

\author{
MORPHOLOGICAL AND QUANTITATIVE EVALUATION OF \\ MUCOUS ACINI OF THE SUBLINGUAL GLAND OF CHRONIC \\ DIABETES AND QUERCETINE SUPPLEMENTED RATS
}

\author{
ALVES, EPB ${ }^{1}$, BORGES, SC ${ }^{1}$, FIORI, JC ${ }^{3}$, LAZARIN, AR $^{3}$, PRADO, MAN $^{3}$, COSTA, $^{2}$ \\ $\mathrm{LH}^{3}$, ALVES, AMP 1 \\ Afiliações: 1- Departamento de Ciências Morfológicas da Universidade Estadual de Maringá. 2- Programa de Pós-Graduação em Ciências \\ Biológicas. Universidade Estadual de Maringá. 3 - Departamento de Medicina da Universidade Estadual de Maringá. \\ Endereço do autor de correspondência: Av. Colombo 5790, Jardim Universitário, Maringá, Paraná, Brasil. CEP: 87020-900. \\ ederpaulo.uem@gmail.com
}

\section{Resumo}

O diabetes crônico suscita diversas alterações morfofuncionais no parênquima das glândulas salivares devido ao aumento do estresse oxidativo e também por complicações metabólicas, vasculares e neuropáticas. Considerando que as glândulas salivares são cruciais para a manutenção da integridade funcional e estrutural da cavidade oral; objetivamos avaliar os parâmetros morfológicos e quantitativos dos ácinos mucosos da glândula sublingual de ratos com diabetes crônico e suplementados com quercetina. Vinte ratos foram distribuídos aleatoriamente em quatro grupos $(n=5)$ : Normoglicêmicos (C); Normoglicêmicos suplementados com quercetina (CQ); Diabéticos (D) e Diabéticos suplementados com quercetina (DQ). Os ratos dos grupos CQ e DQ receberam quercetina (200 mg.Kg-1 de massa corporal) na água, por 120 dias. Os dados obtidos da análise quantitativa não mostrou diferença significativa na densidade acinar entre todos os grupos analisados $(p>0.5)$. A análise morfométrica revelou maior área acinar média nos ratos do grupo CQ em relação aos demais grupos $(\mathbf{p}<0,001)$. Na condição de diabetes, a suplementação com quercetina não alterou os parâmetros morfoquantitativos dos ácinos mucosos destas glândulas. A maior área acinar média observada nos ratos do grupo normoglicêmico suplementado com quercetina (CQ) poderia estar relacionada à capacidade deste antioxidante em regular os processos celulares, metabólicos e de expressão gênica.

Palavras-chave: diabetes mellitus; quercetina; glândula sublingual

\section{Abstract}

Chronic diabetes causes several morphofunctional changes in the parenchyma of the salivary glands due to increased oxidative stress and also due to metabolic, vascular and neuropathic complications. Whereas salivary glands are crucial for maintaining the functional and structural integrity of the oral cavity; we aimed to evaluate the morphological and quantitative parameters of mucous acini of the sublingual gland of rats with chronic diabetes and supplemented with quercetin. Twenty rats were randomly assigned to four groups $(n=5)$ : Normoglycemic $(C)$; Quercetin supplemented normoglycemic (CQ); Diabetics (D) and Quercetin supplemented diabetics (DQ). CQ and DQ rats received quercetin ( $200 \mathrm{mg} . \mathrm{Kg}-1$ body mass) in water for 120 days. The data obtained from the quantitative analysis showed no significant difference in acinar density between all groups analyzed $(p>0.05)$. Morphometric analysis revealed a higher mean acinar area in group QC compared to the other groups $(p<0.001)$. In the condition of diabetes, quercetin supplementation did not alter the morphochemical parameters of the mucous acini of these glands. The largest average acinar area observed in rats from the quercetin-supplemented normoglycemic group (QC) could be related to the ability of this antioxidant to regulate cellular, metabolic and gene expression processes. 


\section{Introdução}

A secreção salivar depende do estado geral de hidratação, mas na prática clínica pode ser bastante afetada por doenças locais ou sistêmicas, tais como Parkinson, hipotireoidismo, depressão, fibrose cística ${ }^{1,2}$, Síndrome de Sjögren ${ }^{3}$, diabetes mellitus (DM) e por vários tipos de medicamentos que causam alterações fisiológicas na cavidade oral $^{5}$, ou ainda pelo processo fisiológico de envelhecimento $^{6}$ que podem culminar em boca seca. Tal condição, pode apresentar-se como um sintoma (xerostomia), um sinal (hipofunção da glândula salivar) ou ambos ${ }^{7}$ Além da xerostomia, outras disfunções na cavidade oral associadas ao diabetes podem ser elencadas: perda dentária, gengivite, periodontite, lesões do tecido mole da língua e mucosa oral ${ }^{1}$. A despeito do parênquima glandular, o diabetes gera alterações morfológicas ${ }^{8}$ e estruturais que são observadas por sialodenites, dobras das lâminas basais de suas células9; perda e atrofia de ácinos ${ }^{10}$; infiltração linfocítica ${ }^{11}$; e aumento de estroma $^{12}$. Em relação à função, as glândulas salivares exibem alterações importantes frente à hiperglicemia crônica. Sob esta condição, têm sido relatadas hipossecreção de enzimas digestivas $^{13}$, alterações na secreção de proteínas ${ }^{14}$ e composição da saliva ${ }^{15}$; acúmulo de lipídeos nas células secretoras; diminuição da resposta à estimulação simpática/parassimpática; aumento da atividade autofágica e lisossomal ${ }^{9}$.

Além das glândulas salivares, as consequências da hiperglicemia a longo prazo, também são observadas em vários órgãos e sistemas corporais, e pode gerar complicações crônicas típicas que culminam em nefropatias, neuropatias, doenças cardiovasculares, disfunções sexuais e gastrointestinais ${ }^{16}$. Estas complicações tem sido atreladas ao aumento do estresse oxidativo, observado no estado diabético $^{17}$.

O tratamento com antioxidantes pode previnir a formação ou neutralizar as espécies radicalares oriundas do estresse oxidativo, minimizando ou evitando as complicações típicas do $\mathrm{DM}^{18,19}$. A quercetina está elencada entre estas substâncias e tem sido amplamente empregada em vários modelos experimentais de diabetes que confirmam a sua atividade antioxidante ${ }^{20}$.

A quercetina $\quad\left(3,5,7,3^{\prime}, \quad 4^{\prime}-\right.$ pentaxidroxiflavona) é a principal representante da subclasse flavonol da família dos flavonoides. 
Esses compostos fenólicos antioxidantes são amplamente consumidos na dieta; podendo estar ligado a açúcares (glicosídeo) ou sem açúcares ligados (aglicona) ${ }^{21}$.

As propriedades antioxidantes da quercetina são elencadas segundo sua atuação: a) inibidora das enzimas ciclooxigenase e lipooxigenase envolvidas na síntese de eicosanóides a partir do ácido araquidônico ${ }^{22}$; b) ação varredora dos ânions superóxido, oxigênio singleto e radicais hidroxila e, consequentemente, prevenção da peroxidação lipídica ${ }^{23}$; c) atuação como agente quelador de íons dos metais de transição como o ferro e o cobre $^{20,24}$, impedindo que estes catalisem reações formadoras de radicais livres ${ }^{25}$.

Considerando que o diabetes mellitus é uma patologia que intensifica o estresse oxidativo e que as glândulas salivares estão entre as estruturas afetadas significativamente por esta condição; e atentando-se ao fato de que alternativas terapêuticas podem ser utilizadas a fim de minimizar as complicações crônicas, proporcionando melhora na qualidade de vida do portador de diabetes; o intento deste estudo foi o de avaliar os parâmetros morfológicos e quantitativos dos ácinos mucosos da glândula sublingual de ratos com diabetes crônico e suplementados com quercetina.

Métodos

\section{Grupo de estudo e delineamento} experimental

Para o presente estudo foram utilizados vinte ratos adultos machos Wistar, provenientes do Biotério Central da Universidade Estadual de Maringá. Aos 88 dias de idade pesando em média $360 \mathrm{~g}$, os animais foram transferidos para o Biotério Setorial do Departamento de Ciências Morfológicas, onde permaneceram alojados em gaiolas individuais providas de bebedouro e ração (NUVILAB ${ }^{\circledR}$ ) ad libitum, mantidos em condições ambientais controladas de temperatura $\left(22^{\circ} \mathrm{C} \pm 2^{\circ} \mathrm{C}\right)$ e ciclo claro/escuro (12/12 horas). Após um período de adaptação ao novo ambiente, os ratos então com 90 dias de idade, foram pesados e disponibilizados para o período experimental de 120 dias. Portanto, os animais foram distribuídos aleatoriamente em quatro grupos contendo 5 (cinco) animais cada, segundo os tratamentos a que foram submetidos, como segue: Grupo C - Animais normoglicêmicos (controle); Grupo CQ Animais normoglicêmicos suplementados com quercetina; Grupo D - Animais diabéticos e 
Grupo DQ - Animais diabéticos suplementados com quercetina. Este estudo teve aprovação pelo Comitê de Ética no Uso de Animais em Experimentação (Parecer do termo de ciência CEUA n $\left.{ }^{\circ} 015 / 2016\right)$.

\section{Indução do diabetes por estreptozootocina}

Para indução do DM, os ratos pertencentes aos grupos D e DQ passaram por um jejum prévio de quatorze horas e receberam injeção endovenosa (veia peniana) de estreptozootocina (STZ) (Sigma, ST. Louis, MO, USA) na dose de $35 \mathrm{mg} / \mathrm{kg}$ de massa corporal, dissolvida em tampão citrato 10 mmol/L (pH 4,5). Após quatro dias da indução, uma gota de sangue foi obtida da cauda dos ratos para mensuração da glicemia pela determinação fotométrica da glicose por meio de glicosocolorante-oxidorredutase (glicosímetro AccuChek Active, Roche Diagnostics GmbH, Mannheim, BW, Alemanha) para confirmar o estabelecimento do DM experimental.

\section{Suplementação com quercetina}

A partir do quarto dia do experimento, após a injeção de estreptozootocina (STZ), os ratos dos grupos CQ e DQ passaram a receber diariamente água suplementada com quercetina na dosagem de $200 \mathrm{mg} \cdot \mathrm{Kg}^{-1}$ de massa corporal, sendo realizada uma avaliação periódica da ingestão de água e peso destes animais para manutenção desta dosagem. Os animais dos grupos $\mathrm{C}$ e $\mathrm{D}$ receberam água sem suplementação.

\section{Coleta e processamento do material}

Após 120 dias de experimento, os ratos (com 210 dias de idade) foram eutanasiados, sendo previamente pesados e anestesiados com uma dose de $40 \mathrm{mg} \cdot \mathrm{kg}^{-1}$ de massa corporal de Tiopental® (Laboratórios Abbott, Chicago, IL, EUA) intraperitoneal. O sangue foi coletado por punção cardíaca para avaliação da glicemia sanguínea pelo método da glicose oxidase.

As glândulas extraídas foram lavadas em solução salina $(\mathrm{NaCl}$ 0,9\%) para retirada de resíduos de sangue, e imediatamente transferidas para solução de formalina $10 \%$ por um período de 12 horas, promovendo a íntegra fixação dos tecidos glandulares. Sequencialmente, as mesmas foram lavadas em água corrente para serem armazenadas em álcool 70\%. Utilizandose uma série crescente de concentrações de alcoóis, os tecidos foram desidratados, diafanizados em xilol, incluídos em parafina e 
seccionados em micrótomo com $6 \mu \mathrm{m}$ espessura.

Posteriormente, os cortes foram submetidos à

coloração em hematoxilina-eosina (HE).

Análise morfométrica dos ácinos

glandulares

As imagens dos ácinos foram capturadas por câmara de alta resolução acoplada ao microscópio Olympus BX40, e transmitidas para microcomputador e gravadas em compact disc.

Por meio do programa computadorizado de análise de imagens Image-Pro-Plus, foi mensurada a área $\left(\mu \mathrm{m}^{2}\right)$ do perfil de 100 ácinos por lâmina, perfazendo o total de 500 ácinos em cada grupo estudado, expressando-se os resultados em média das áreas do ácinos por grupo.

\section{Análise quantitativa dos ácinos}

\section{glandulares}

As imagens dos ácinos foram capturadas por câmara de alta resolução acoplada ao microscópio Olympus BX40, e transmitidas para microcomputador e gravadas em compact disc. Por meio do programa computadorizado de análise de imagens Image-Pro-Plus, foram quantificados todos os ácinos de 20 imagens capturadas por animal em aumento de 20X, com a finalidade de delinear a densidade destas estruturas.

\section{Análise estatística}

Os dados coletados foram submetidos a testes estatísticos apropriados, como análise de variância e pós-teste de Tukey para comparação dos aspectos morfoquantitativos dos ácinos glandulares entre os grupos. O nível de significância foi de $5 \%$.

\section{Resultados}

A análise morfométrica dos ácinos mucosos glandulares (Figura 1) revelou maior área acinar média nos animais do grupo CQ em relação aos demais grupos $(\mathrm{p}<0,001)$. Além disso, não foi observada diferença significativa entre as áreas acinares médias dos grupos C, D e DQ $(p>0,05)$.

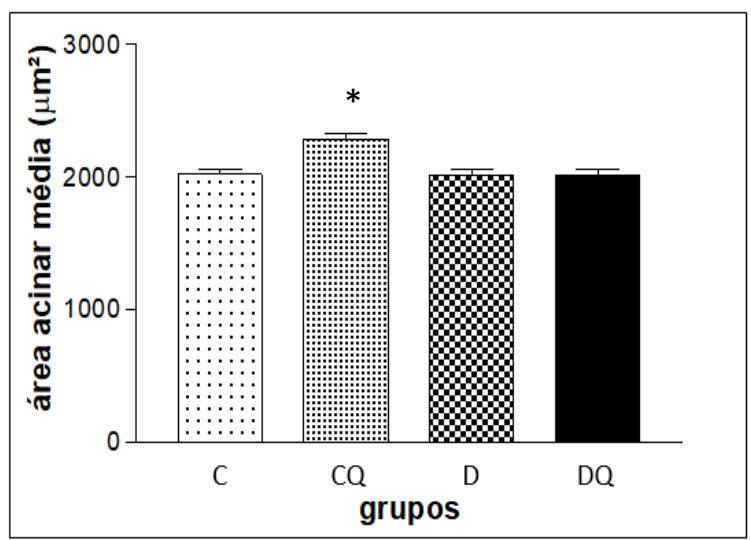

Figura 1. Área acinar média da glândula salivar sublingual dos ratos nos grupos: (C) normoglicêmicos; (CQ) normoglicêmicos suplementados com quercetina; (D) diabéticos e (DQ) diabéticos suplementados com quercetina $\mathrm{N}=5$. * $\mathrm{p}<0,001$ quando comparado aos grupos $\mathrm{C}, \mathrm{D}$ e DQ. 
Os resultados obtidos através da análise quantitativa dos ácinos mucosos (Figura 2) da glândula sublingual não revelaram diferença significativa entre todos os grupos analisados (p>0.05). Na Figura 3 são apresentados os parênquimas da glândula salivar sublingual de ratos normoglicêmicos (C), normoglicêmicos suplementados com quercetina (CQ); diabéticos (D) e diabéticos suplementados com quercetina (DQ).

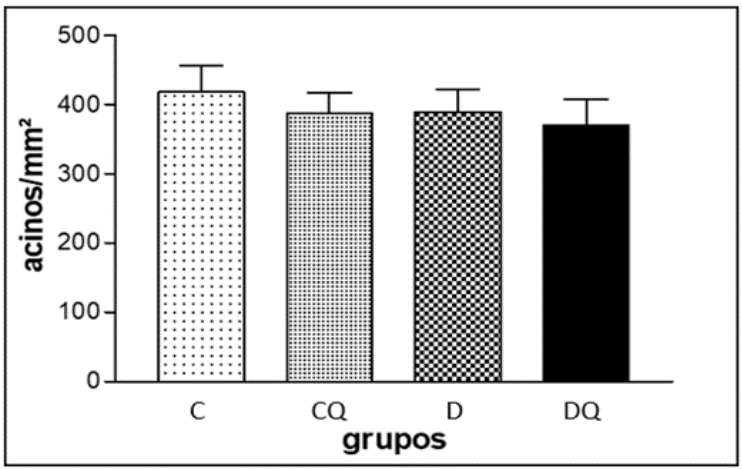

Figura 2 - Densidade acinar média dos acinos mucosos da glândula sublingual dos ratos nos grupos: Normoglicêmicos (C); Normoglicêmicos suplementados com quercetina (CQ); Diabéticos (D); Diabéticos suplementados com quercetina (DQ). N=5. p>0.05 entre todos os grupos analisados.

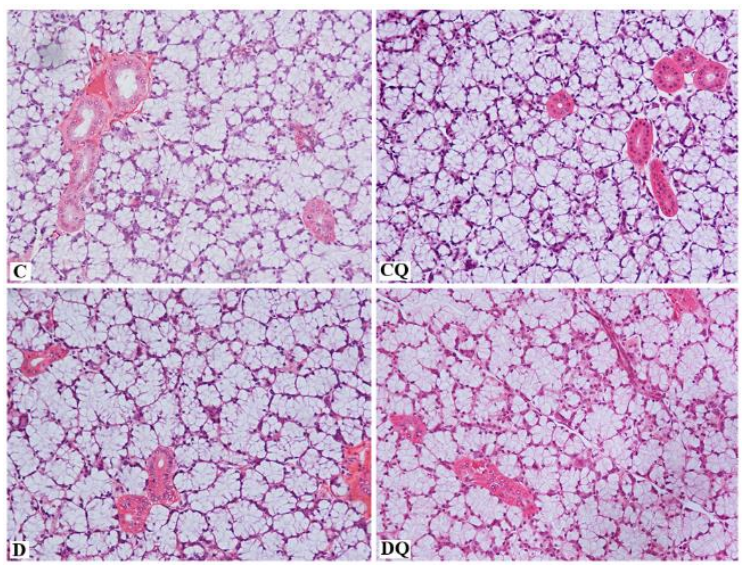

Figura 3 - Parênquima da glândula sublingual corado com Hematoxilina e Eosina (HE) dos grupos normoglicêmico/controle (C), normoglicêmico suplementado com quercetina (CQ), diabéticos (D) e diabéticos suplementados com quercetina (DQ) obtidas com microscópio óptico Olympus e objetiva de $20 \mathrm{X}$.

\section{Discussão}

A perda numérica de ácinos das glândulas salivares de animais com diabetes crônico é frequentemente reportada; sobretudo na glândula parótida ${ }^{26}$. Alguns fatores podem ser apontados para a perda acinar desta glândula, e entre eles estão, a associação entre o estado hiperglicêmico e aumento do estresse oxidativo com concomitante redução na capacidade antioxidante $^{27}$; a auto-oxidação da glicose, glicosilação protéica, peroxidação lipídica e formação de produtos de glicosilação avançadas $(\mathrm{AGEs})^{28}$. Devido à intensa ação das espécies radicalares, as células denotam debilitada capacidade em neutralizá-las e eliminá-las; fato este, que pode gerar morte das mesmas por necrose ou apoptose ${ }^{29}$. Outra condição observada, é o aumento da infiltração de linfócitos no parênquima glandular, que em virtude do processo inflamatório gerado, incorre em destruição tecidual ${ }^{30}$.

Os estudos clínicos e experimentais que versam sobre os efeitos prejudiciais do diabetes nas glândulas salivares são em sua maioria relacionados às glândulas parótidas e submandibulares; uma vez que as glândulas sublinguais, por contribuírem com apenas $5 \%$ da produção de saliva $^{31}$, são menos avaliadas, de tal 
modo que a literatura apresenta raríssimos dados histopatológicos sobre a glândula sublingual na condição de diabetes. Além disso, entre as três glândulas salivares maiores, observa-se que as complicações características do diabetes, como o estresse oxidativo, são mais evidenciadas na glândula parótida do que nas outras duas ${ }^{26}$.

Os resultados obtidos em nosso estudo, corroboram com os achados de Morris et al. ${ }^{32}$, os quais não constataram alterações morfológicas nas células parenquimais da glândula sublingual de ratos diabéticos induzidos por estreptozootocina. Apesar de não elucidadas as causas desta ação diferencial do diabetes sobre o parênquima das diferentes glândulas, há de se considerar que alguns fatores sejam atribuídos à tal suscetibilidade, tais como: 1) constituintes de membrana celular ou composição da secreção que apresentam diferente sensibilidade de resposta à glicose circulante; 2) variações no suprimento sanguíneo devido às alterações vasculares; 3) ação dos receptores de insulina que estariam ligados à modificação da sensibilidade celular; 4) vulnerabilidade a alterações metabólicas de cada glândula $\left.{ }^{8} ; 5\right)$ danos estruturais nos nervos autonômicos ${ }^{33}$.

Embora nossos dados não tenham evidenciado os potenciais efeitos da quercetina sobre a densidade acinar durante o período do quadro hiperglicêmico; a literatura indica vastas diligências promissoras deste antioxidante em outros órgãos e sistemas corporais em diferentes condições patológicas, tais como: antihipertensiva $^{34}$, antiarítmica, antihepatoxicicidade ${ }^{35}$, hipocolesterolêmica ${ }^{20}$, anticarcinogênica $^{36}$, antiviral, antiulcerogênica, antitrombótica, anti-isquêmica, anti-inflamatória e antialérgica ${ }^{22,37}$. Além disso, em alguns estudos são evidenciados os efeitos benéficos de outros antioxidantes sobre o parênquima glandular nas glândulas parótidas de ratos diabéticos suplementados com resveratrol ${ }^{10}$, com vitaminas C e $E^{38}$, e em glândulas submandibulares de camundongos submetidos à radiação e tratados com vitamina $\mathrm{E}^{39}$.

As glândulas salivares de humanos e várias espécies animais apresentam graus distintos de alterações morfofisiológicas e estruturais, dependendo de fatores como o estado fisiológico de envelhecimento ${ }^{40}$, de patologias como a Síndrome de Sjögren $^{41}$ e diabetes mellitus ${ }^{13}$. No entanto, tem sido observado que estas glândulas também apresentam maior ou menor sensibilidade às injúrias causadas por estes fatores supracitados, sobretudo quando se trata de diabetes. 
Em ratos com diabetes crônico, a atrofia acinar tem sido correlacionada à desidratação celular, em virtude do esgotamento crônico da concentração de sódio nestas células; à modificação no conteúdo proteico e comprometimento da síntese de proteínas ${ }^{9,42}$ e no metabolismo de carboidratos; bem como à redução da resposta secretória das glândulas salivares às estimulações simpática e parassimpática devido à neuropatia diabética ${ }^{33} \mathrm{e}$ à insuficiência ou ausência de insulina, que compromete a manutenção da estrutura e função normal da glândula ${ }^{43}$.

$$
\text { A glândula sublingual é menos }
$$
vulnerável aos danos celulares provenientes do DM, de modo que seu parênquima não parece ser afetado morfologicamente. No entanto, a longo prazo, gotículas de lipídeos vão se formando no citoplasma das células acinares, contribuindo com a disfunção acinar ${ }^{44}$. Em nosso estudo, a análise morfométrica (Figura 1) não indicou nenhum tipo de alteração morfológica dos ácinos mucosos nos ratos diabéticos (D), quando comparados aos do grupo controle (C), e a suplementação com quercetina nos ratos diabéticos (DQ) também não interferiu nesse quesito. A maior área acinar média observada no grupo (CQ), provavelmente esteja atrelada ao papel deste flavonóide na regulação do metabolismo celular e expressão gênica ${ }^{45}$, além de aumentar a expressão de aquaporina 5 (AQP5) e a absorção de cálcio que estimula a secreção de saliva ${ }^{39}$. Além disso, nestes ratos, a presença da insulina gera o transporte de muitos aminoácidos para dentro das células, além de atuar diretamente sobre os ribossomas aumentando a tradução de RNAm formando assim novas proteínas ${ }^{46}$.

\section{Conclusão}

O Diabetes Mellitus (DM) não alterou a densidade e a morfometria acinar, e a suplementação com quercetina também não exibiu nenhum efeito sobre o parênquima destas glândulas. A maior área acinar média observada nos ratos do grupo normoglicêmico suplementado com quercetina (CQ) poderia estar relacionada à capacidade deste antioxidante em regular os processos celulares, metabólicos e de expressão gênica, contribuindo para o aperfeiçoamento destes parâmetros durante o processo fisiológico normal de envelhecimento.

\section{Referências}

1 Moore PA, Guggenheimer J, Etzel KR, Weyant RJ, Orchard T. Type 1 diabetes mellitus, 
xerostomia, and salivary flow rates. Oral Surg Oral Med Oral Pathol Oral Radiol Endod 2001. doi:10.1067/moe.2001.117815.

2 Alves CDAD, Aguiar RA, Alves ACS, Santana MA. Diabetes melito: Uma importante co-morbidade da fibrose cística. J. Bras. Pneumol. 2007. doi:10.1590/s180637132007000200017.

3 Rusthen S, Young A, Herlofson BB, Aqrawi LA, Rykke M, Hove LH et al. Oral disorders, saliva secretion, and oral healthrelated quality of life in patients with primary Sjögren's syndrome. Eur J Oral Sci 2017. doi:10.1111/eos.12358.

4 López-Pintor RM, Casañas E, GonzálezSerrano J, Serrano J, Ramírez L, De Arriba L et al. Xerostomia, Hyposalivation, and Salivary Flow in Diabetes Patients. J. Diabetes Res. 2016. doi:10.1155/2016/4372852.

5 Okamoto A, Miyachi H, Tanaka K, Chikazu D, Miyaoka H. Relationship between xerostomia and psychotropic drugs in patients with schizophrenia: evaluation using an oral moisture meter. J Clin Pharm Ther 2016. doi:10.1111/jcpt.12449.

6 Gomes LF, Fernanda SI, Lopes F. Salivary flow and xerostomia in premenopausal and postmenopausal women (Estudo sobre o fluxo salivar e xerostomia em mulheres na pré e pós-menopausa). Pesqui Bras Odontopediatria Clin Integr 2007.

$7 \quad$ Frydrych AM. Dry mouth: Xerostomia and salivary gland hypofunction. Aust Fam Physician 2016.

8 Lilliu MA, Solinas P, Cossu M, Puxeddu $\mathrm{R}$, Loy $\mathrm{F}$, Isola $\mathrm{R}$ et al. Diabetes causes morphological changes in human submandibular gland: A morphometric study. J Oral Pathol Med 2015. doi:10.1111/jop.12238.

9 Mednieks MI, Szczepanski A, Clark B, Hand AR. Protein expression in salivary glands of rats with streptozotocin diabetes. Int J Exp Pathol 2009. doi:10.1111/j.13652613.2009.00662.x.

10 Pereira Alves ${ }^{1}$ AM, Cristina Buttow N, Christmann C, Yamamoto E, Medeiros J, Mello
DE et al. Resveratrol atenua a atrofia e a perda de ácinos da glândula salivar parótida de ratos com diabetes crônico resveratrol reduces losses and atrophy of acinar cells of parotid gland of diabetic rats. 2017 ; 30: 6-10.

11 Markopoulos AK, Belazi M. Histopathological and immunohistochemical features of the labial salivary glands in children with type I diabetes. J Diabetes Complications 1998. doi:10.1016/S1056-8727(97)00047-0.

12 Carda C, Mosquera-Lloreda N, Salom L, Gomez De Ferraris ME, Peydró A. A structural and functional salivary disorders in type 2 diabetic patients. Med Oral Patol Oral Cir Bucal 2006.

13 Mahay S, Adeghate E, Lindley MZ, Rolph CE, Singh J. Streptozotocin-induced type 1 diabetes mellitus alters the morphology, secretory function and acyl lipid contents in the isolated rat parotid salivary gland. Mol Cell Biochem 2004. doi:10.1023/B:MCBI.0000028753.33225.68.

14 Isola M, Cossu M, Diana M, Isola R, Loy F, Solinas P et al. Diabetes reduces statherin in human parotid: Immunogold study and comparison with submandibular gland. Oral Dis 2012. doi:10.1111/j.1601-0825.2011.01884.x.

15 Negrato C, Tarzia O. Buccal alterations in diabetes mellitus. Diabetol. Metab. Syndr. 2010. doi:10.1186/1758-5996-2-3.

16 Alberti KGMM, Zimmet PZ. Definition, diagnosis and classification of diabetes mellitus and its complications. Part 1: Diagnosis and classification of diabetes mellitus. Provisional report of a WHO consultation. Diabet Med 1998. doi:10.1002/(SICI)1096-

9136(199807)15:7<539::AID-

DIA668>3.0.CO;2-S.

17 Evans JL, Goldfine ID, Maddux BA, Grodsky GM. Oxidative stress and stressactivated signaling pathways: A unifying hypothesis of type 2 diabetes. Endocr Rev 2002. doi:10.1210/er.2001-0039.

18 Shirpoor A, Khadem Ansari MH, Salami S, Ghaderi Pakdel F, Rasmi Y. Effect of vitamin $\mathrm{E}$ on oxidative stress status in small intestine of diabetic rat. World J Gastroenterol 2007. 
doi:10.3748/wjg.v13.i32.4340.

19 Zhu H, Wang Y, Liu Z, Wang J, Wan D, Feng $S$ et al. Antidiabetic and antioxidant effects of catalpol extracted from Rehmannia glutinosa (Di Huang) on rat diabetes induced by streptozotocin and high-fat, high-sugar feed. Chinese Med (United Kingdom) 2016. doi:10.1186/s13020-016-0096-7.

20 Formica J V., Regelson W. Review of the biology of quercetin and related bioflavonoids. Food Chem. Toxicol. 1995. doi:10.1016/0278-6915(95)00077-1.

21 Ulusoy HG, Sanlier N. A minireview of quercetin: from its metabolism to possible mechanisms of its biological activities. Crit. Rev. Food Sci. Nutr. 2019. doi:10.1080/10408398.2019.1683810.

22 Erden Inal M, Kahraman A. The protective effect of flavonol quercetin against ultraviolet a induced oxidative stress in rats. Toxicology 2000. doi:10.1016/S0300483X(00)00268-7.

23 Hollman PCH, Katan MB. Absorption, metabolism and health effects of dietary flavonoids in man. Biomed Pharmacother 1997. doi:10.1016/S0753-3322(97)88045-6.

24 Morel I, Lescoat G, Cogrel P, Sergent O, Pasdeloup N, Brissot P et al. Antioxidant and iron-chelating activities of the flavonoids catechin, quercetin and diosmetin on iron-loaded rat hepatocyte cultures. Biochem Pharmacol 1993. doi:10.1016/0006-2952(93)90371-3.

25 Rigo A, Guterres S. O potencial antioxidante de vegetais no combate ao envelhecimento cutâneo. Infarma 2002; 14: 6973.

26 Knaś M, Maciejczyk M, Daniszewska I, Klimiuk A, Matczuk J, Kołodziej $U$ et al. Oxidative Damage to the Salivary Glands of Rats with Streptozotocin-Induced DiabetesTemporal Study: Oxidative Stress and Diabetic Salivary Glands. J Diabetes Res 2016. doi:10.1155/2016/4583742.

27 Bonnefont-Rousselot D. Glucose and reactive oxygen species. Curr. Opin. Clin. Nutr. Metab. Care. 2002. doi:10.1097/00075197-
200209000-00016.

28 Ighodaro OM. Molecular pathways associated with oxidative stress in diabetes mellitus. Biomed. Pharmacother. 2018. doi:10.1016/j.biopha.2018.09.058.

29 Obrosova IG, Van Huysen C, Fathallah L, Cao XC, Greene DA, Stevens MJ. An aldose reductase inhibitor reverses early diabetesinduced changes in peripheral nerve function, metabolism, and antioxidative defense. FASEB J 2002. doi:10.1096/fj.01-0603fje.

30 Moreira CR, Ferrari F, Alves ÉPB, Bin L, Machado J, Dalzotto $E$ et al. Infiltração Linfocítica no Parênquima da Glândula Salivar Parótida de Ratos Diabéticos Suplementados com Acetil-L-Carnitina. Saúde e Pesqui 2014.

31 Arana V, Katchburian E. Histologia e Embriologia Oral - Texto, Atlas, Correlações Clínicas. 3rd ed. Rio de Janeiro, 2012.

32 Morris PA, Prout RES, Proctor GB, Garrett JR, Anderson LC. Lipid analysis of the major salivary glands in streptozotocin-diabetic rats and the effects of insulin treatment. Arch Oral Biol 1992. doi:10.1016/00039969(92)90105-H.

33 Anderson LC, Garrett JR, Thulin A, Proctor GB. Effects of streptozocin-induced diabetes on sympathetic and parasympathetic stimulation of parotid salivary gland function in rats. Diabetes 1989. doi:10.2337/diab.38.11.1381.

34 Marunaka Y, Marunaka R, Sun H, Yamamoto T, Kanamura N, Inui $\mathrm{T}$ et al. Actions of quercetin, a polyphenol, on blood pressure. Molecules. 2017. doi:10.3390/molecules22020209.

35 Miltonprabu S, Tomczyk M, SkalickaWoźniak K, Rastrelli L, Daglia M, Nabavi SF et al. Hepatoprotective effect of quercetin: From chemistry to medicine. Food Chem Toxicol 2017. doi:10.1016/j.fct.2016.08.034.

36 Rauf A, Imran M, Khan IA, ur-Rehman M, Gilani SA, Mehmood Z et al. Anticancer potential of quercetin: A comprehensive review. Phyther. Res. 2018. doi:10.1002/ptr.6155. 
37 Shirai M, Yamanishi R, Moon JH, Murota K, Terao J. Effect of quercetin and its conjugated metabolite on the hydrogen peroxide-induced intracellular production of reactive oxygen species in mouse fibroblasts. Biosci Biotechnol Biochem 2002. doi:10.1271/bbb.66.1015.

38 Alves ÂMP, Alves ÉPB, De Mello JM, Zanoni JN, Bespalhok DDN, Moreira CR et al. Avaliação dos efeitos da suplementação com vitaminas $\mathrm{E}$ e C sobre os ácinos da glândula parótida de ratos diabéticos crônicos: análise morfológica e quantitativa. Rev Bras Ciências da Saúde - USCS 2016. doi:10.13037/ras.vol14n47.3533.

39 Takahashi A, Inoue $\mathrm{H}$, Mishima $\mathrm{K}$, Ide F, Nakayama R, Hasaka A et al. Evaluation of the effects of quercetin on damaged salivary secretion. PLoS One 2015. doi:10.1371/journal.pone.0116008.

40 Li L, Wang H, Hu L, Wu X, Zhao B, Fan $\mathrm{Z}$ et al. Age associated decrease of sialin in salivary glands. Biotech Histochem 2018. doi:10.1080/10520295.2018.1463453.

41 Atkinson JC, Travis WD, Pillemer SR, Bermudez D, Wolff A, Fox PC. Major salivary gland function in primary Sjogren's syndrome and its relationship to clinical features. J Rheumatol 1990.

42 Guyton A, Hall J. Tratado de fisiologia médica. 13th ed. Rio de Janeiro, 2017.

43 Hand AR, Weiss RE. Effects of streptozotocin-induced diabetes on the rat parotid gland. Lab Investig 1984.

44 Kamata M, Shirakawa M, Kikuchi K, Matsuoka T, Aiyama S. Histological analysis of the sublingual gland in rats with streptozotocininduced diabetes. Okajimas Folia Anat Jpn 2007. doi:10.2535/ofaj.84.71.

45 Tarahovsky YS, Kim YA, Yagolnik EA, Muzafarov EN. Flavonoid-membrane interactions: Involvement of flavonoid-metal complexes in raft signaling. Biochim. Biophys. Acta - $\quad$ Biomembr. 2014. doi:10.1016/j.bbamem.2014.01.021.

46 Kim SK, Cuzzort LM, Mckean RK, Allen ED. Effects of Diabetes and Insulin on $\alpha$ amylase Messenger RNA Levels in Rat Parotid Glands. J Dent Res 1990. doi:10.1177/00220345900690081001.

\section{Agradecimentos}

Os autores agradecem Maria dos Anjos Fortunato e Maria Angela Moreira da Costa por seu valioso suporte técnico.

Data de submissão: 26/11/2019

Data de aprovação: 13/12/2019 